\title{
REVIEW \\ Bladder cancer in individuals with spinal cord injuries: a meta-analysis
}

\author{
L Gui-Zhong and M Li-Bo
}

Objectives: To conduct a scoping review of all the literature related to bladder cancer in individuals with spinal cord injuries (SCIs). Methods: We searched for trials in MEDLINE, EMBASE, CINAHL and the Cochrane Register. Meta-analysis methods were used to evaluate these studies.

Results: A total of 18 articles were identified and included as the evidence base for analysis. The incidence of bladder cancer in individuals with $\mathrm{SCl}$ is $6 \%$ (95\% confidence interval $(\mathrm{Cl}), 5-6 \%, R=49.2 \%)$. Mean patient age at bladder cancer diagnosis was 50 years $(95 \% \mathrm{Cl}, 45-55$ years, $R=92.7 \%)$. Length of indwelling catheterization of the patient population ranged from 6 to 29 years, with an average of 16 years $(95 \% \mathrm{Cl}, 12-20$ years, $R=94.4 \%)$. The interval between $\mathrm{SCl}$ and the first diagnosis of bladder cancer was 24 years $(95 \% \mathrm{Cl}, 21-27$ years, $R=85.3 \%)$. In the $\mathrm{SCl}$ population the estimated percent of squamous cell carcinoma is $36.8 \%(95 \%$ $\left.\mathrm{Cl}, 31.6-42.5 \%, R^{2}=32.3 \%\right)$. In addition, $46.3 \%\left(95 \% \mathrm{Cl}, 33.7-59.4 \%, \mathrm{I}^{2}=40.5 \%\right)$ of the bladder cancer histology was traditional transitional cell carcinoma. Other pathological subtypes account for $17.1 \%(95 \% \mathrm{Cl}, 12.8-22.6 \%, P=21.5 \%)$. The 1 -year overall survival rate after treatment of bladder cancer was $62.1 \%(95 \% \mathrm{Cl}, 56.2-66.7 \%, R=21.3 \%)$. Cystoscopy demonstrated a sensitivity of $64 \%(95 \% \mathrm{Cl}, 49.3-76.5 \%, R=37.7 \%)$ for detecting bladder cancer. Cytology demonstrated a sensitivity of $36.3 \%(95 \% \mathrm{Cl}$, $21.5-54.3 \%, R=40.2 \%)$ for detecting bladder cancer.

Conclusion: Bladder cancer is a rare and potentially lethal occurrence in $\mathrm{SCl}$ patients. Physicians need to have a high index of suspicion for bladder cancer, particularly among SCl patients managed with long-term indwelling catheters.

Spinal Cord (2017) 55, 341-345; doi:10.1038/sc.2016.151; published online 8 November 2016

\section{INTRODUCTION}

Bladder cancer is a relatively rare disorder among the general population, with a global age-standardized incidence of 9.0 for men and 2.2 to 100000 for women on average in the world in the year 2012. ${ }^{1}$ Of the bladder cancer, $90 \%$ is transitional cell carcinoma (TCC), and the remaining $10 \%$ consists of squamous cell carcinoma (SCC), adenocarcinoma and other rare subtypes. ${ }^{2}$ Bladder cancer specifically is the third leading cause of cancer death in spinal cord injury (SCI) population, whereas it is ranked tenth in the general population. ${ }^{3}$ The current state of evidence surrounding bladder cancer epidemiology, risk factors, presentation, screening and treatment among SCI patients has not been reviewed. The objective of this study was to conduct a scoping review and summarize the current state of knowledge, and suggest areas for future research.

\section{MATERIALS AND METHODS}

A computer-assisted search of multiple databases (PubMed, CINAHL and the Cochrane Register) was conducted for experimental and clinical literature up to and including June 2015. Our search strategy was based on the preselected keywords or MeSH headings (spinal cord injury, neurogenic bladder, bladder cancer, TCC, urothelial carcinoma, squamous cell carcinoma). Keywords were used in various combinations to maximize search results. The inclusion criteria were any article primarily reporting on an aspect of bladder cancer among SCI patients (based on a review of the article title and abstract, and where necessary full text). This primarily included articles related to the epidemiology, risk factors, screening, treatment and outcomes of bladder cancer in SCI patients. Our only exclusion criterion was non-English language publications; given the limited literature base, no studies were excluded on the basis of publication date or research methodology. The indexing of identified articles within their identified database was reviewed to ensure other relevant indexing terms had not been missed. The reference lists from articles identified in the computer searches were examined to identify potentially relevant investigations. The method of citation tracking was used to further identify authors who published potentially relevant studies. Individual articles selected for review were scanned for additional citations not identified by the original search.

We used $I^{2}$ to assess heterogeneity between studies, using fixed effects models where there was low heterogeneity $\left(I^{2}<50 \%\right)$, otherwise using random effects models. In statistics, a random effect (s) model is a kind of hierarchical linear model. It assumes that the data set being analyzed consists of a hierarchy of different populations whose differences relate to that hierarchy. The random effects model is a special case of the fixed effects model. In biostatistics, biostatisticians use 'fixed' and 'random' effects to respectively refer to the population-average and subject-specific effects (and where the latter are generally assumed to be unknown, latent variables). We used the 'metabin' function of the 'meta' package in MetaAnalyst Beta 2.13 (a software program, Tufts Medical Center, Boston, MA, USA). We used meta-analysis regression, with inverse variance weighting and DerSimonian-Laird. The analyzed studies are not weighted. 
This study is an analysis of published data and does not require ethics committee approval.

\section{RESULTS}

The search yielded a total of 89 non-overlapping reports that were broadly contributed as potentially relevant to bladder cancer in individuals with SCIs. The abstracts of all studies were initially examined by one author (LG-Z) to determine if they met basic inclusion criteria. Forty-eight studies were eliminated after review of the abstract. The full reports for the remaining 41 studies were examined by two raters (LG-Z and $\mathrm{ML}$ ) to determine the appropriateness of the study for further analysis. An additional 23 studies were eliminated following examination of the complete articles, leaving 18 studies for analysis.

The incidence of bladder cancer in individuals with SCI is $6 \%$ (95\% confidence interval (CI), 5-6\%,$I^{2}=49.2 \%$, Figure 1). Mean patient age at bladder cancer diagnosis was 50 years $(95 \%$ CI, 45-55 years, $I^{2}=92.7 \%$, Figure 2). Length of indwelling catheterization of the patient population ranged from 6 to 29 years, with an average of 16 years (95\% CI, $12-20$ years, $I^{2}=94.4 \%$, Figure 3 ). A majority of studies report a consistent mean of 18-34 years from the onset of the SCI to the diagnosis of bladder cancer. The interval between SCI and the first diagnosis of bladder cancer was 24 years (95\% CI, 21-27 years, $I^{2}=85.3 \%$, Figure 4 ). Bladder cancer within SCI patients also appears to follow a different tumor subtype. In the SCI population the estimated percent of SCC is $36.8 \%$ (95\% CI, 31.6-42.5\%, $I^{2}=32.3 \%$, Figure 5). In addition, $46.3 \%$ (95\% CI, 33.7-59.4\%, $I^{2}=40.5 \%$, Figure 6) of the bladder cancer histology was traditional TCC. Other pathological subtypes account for $17.1 \%$ (95\% CI, $12.8-22.6 \%$, $I^{2}=21.5 \%$, Figure 7 ). The 1 -year overall survival rate after treatment of bladder cancer was $62.1 \%$ (95\% CI, $56.2-66.7 \%, I^{2}=21.3 \%$, Figure 8). Cystoscopy demonstrated a sensitivity of $64 \%$ (95\% CI, $49.3-76.5 \% I^{2}=37.7 \%$, Figure 9) for detecting bladder cancer. Cytology demonstrated a sensitivity of $36.3 \%$ (95\% CI, 21.5-54.3\%, $I^{2}=40.2 \%$, Figure 10) for detecting bladder cancer.

\section{DISCUSSION}

Multiple case series have been published examining the relationship between bladder cancer and SCI patients; they have reported simple incidences of $0.1-10 \% .^{4-18}$ In this study, the pooled incidence of bladder cancer in individuals with SCI is 6\%o (95\% CI, 5-6\%o). The variable-reported incidence of bladder cancer in individuals with SCI is likely because of several factors: different mean ages, risk factors (indwelling foley catheters, type of catheter material, smoking), screening protocols, referral patterns, statistical methods and improved survival of SCI patients over time. Determining an accurate and modern estimation of incidence in relation to the general population is an important step in assessing the risk of bladder cancer among current SCI patients. ${ }^{19}$

A mean age of bladder cancer diagnosis was 50 years ( $95 \%$ CI, 45-55 years old). In the general population the average age at bladder cancer diagnosis is 73 years, with the highest percentage

\section{Forest Plot: $\mathbf{9 5 \%}$ Confidence Interval}

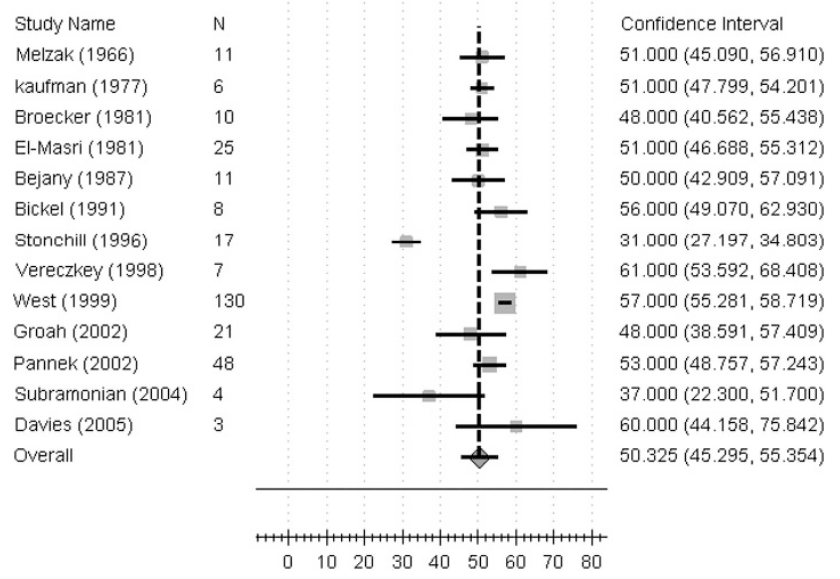

Figure 2 The mean age of patients of bladder cancer meta-analysis. A full color version of this figure is available at the Spinal Cord journal online.

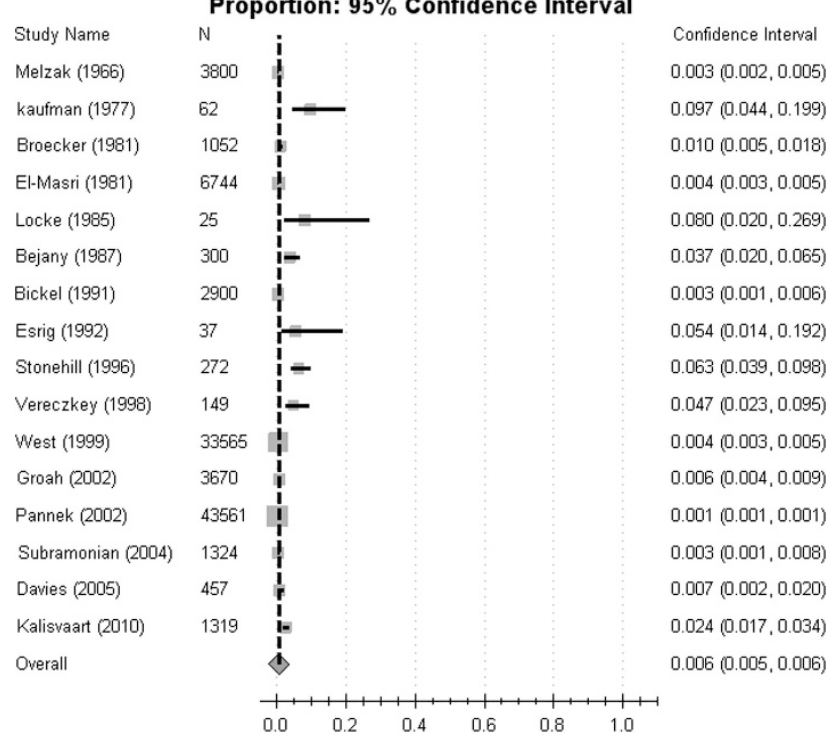

Figure 1 The incidence of bladder cancer in $\mathrm{SCl}$ patient meta-analysis. A full color version of this figure is available at the Spinal Cord journal online.

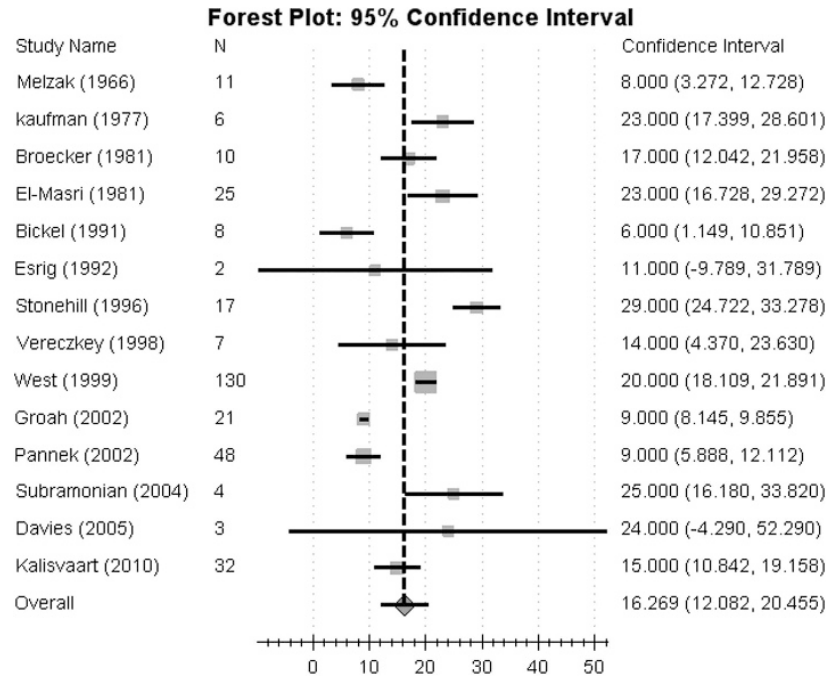

Figure 3 Length of indwelling catheterization in individuals with bladder cancer meta-analysis. A full color version of this figure is available at the Spinal Cord journal online. 
Forest Plot: $\mathbf{9 5} \%$ Confidence Interval

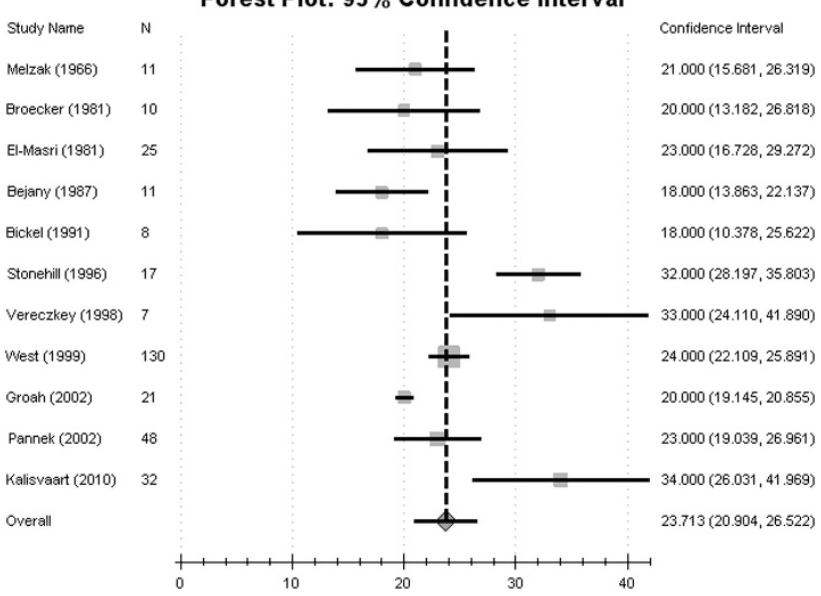

Figure 4 The interval between $\mathrm{SCl}$ and the first diagnosis of bladder cancer meta-analysis. A full color version of this figure is available at the Spinal Cord journal online.

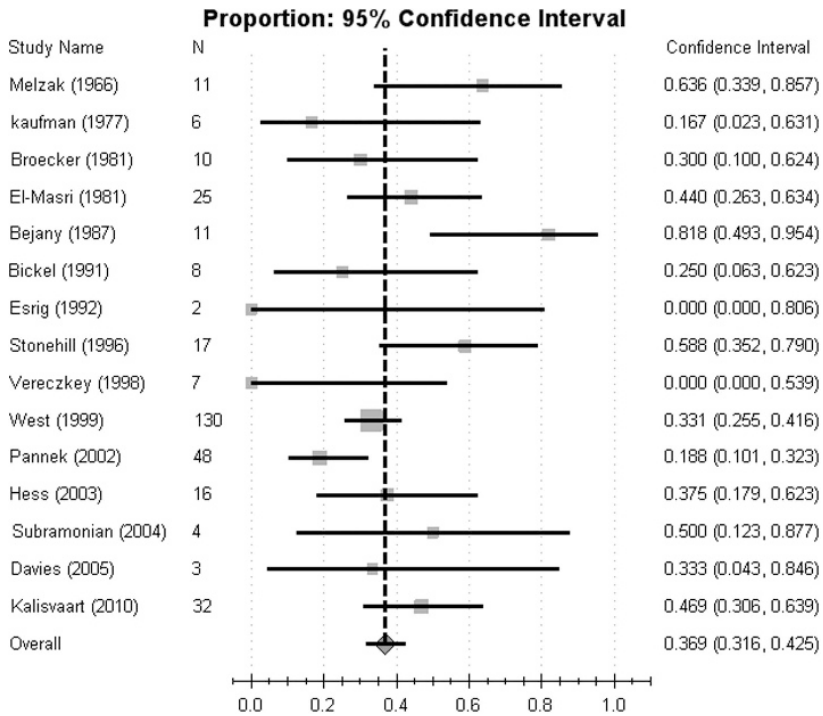

Figure 5 The percent of SCC in the SCl population meta-analysis. A full color version of this figure is available at the Spinal Cord journal online.

between ages 75 and 84 years. ${ }^{3}$ Prior studies found that bladder cancer develops in people with SCI earlier than normal, at a mean age of 48 to 61 years, ${ }^{19}$ and $\sim 24 \%$ were diagnosed at $<40$ years of age. ${ }^{7}$ The finding of Nahm et al. ${ }^{20}$ of a standardized mortality ratio of 20 in patients with SCI aged 30 to 59 years is largely consistent with previous studies, and suggests that patients with SCI are exposed to risk factors earlier or to risks that are more pernicious.

There appears to be a consistent relationship between the time of the SCI and the diagnosis of bladder cancer. The majority of studies report a consistent mean of 18-34 years from the onset of the SCI to the diagnosis of bladder cancer. ${ }^{6-18}$ The interval between SCI and the first diagnosis of bladder cancer was 24 years (95\% CI, 21-27 years). The prolonged time lapse between SCI and bladder cancer may be the result of a generally young population at the time of SCI who would be unlikely to develop bladder cancer. However, the younger than expected mean age of diagnosis suggests there is an

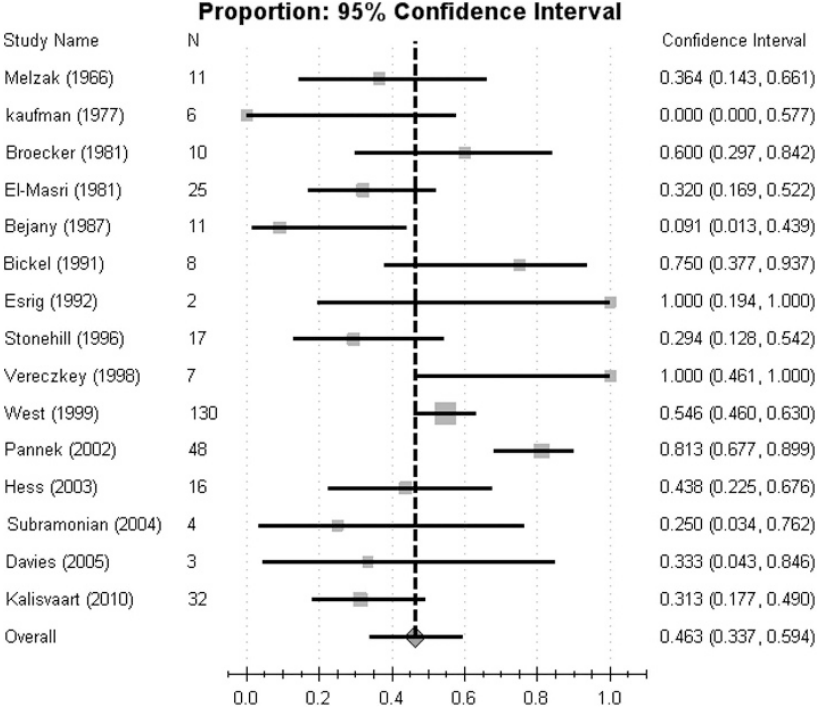

Figure 6 The percent of TCC in the SCl population meta-analysis. A full color version of this figure is available at the Spinal Cord journal online.

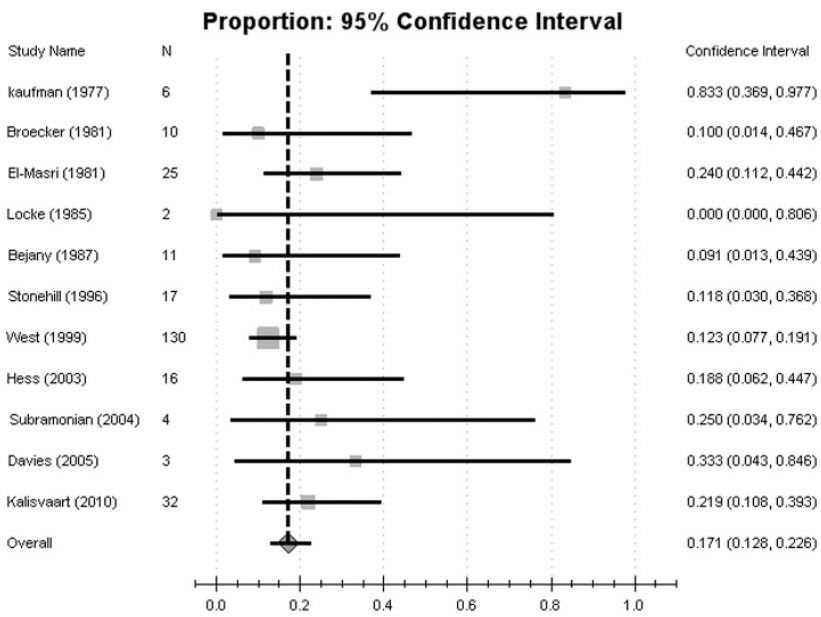

Figure 7 The percent of other subtypes of bladder cancer in the $\mathrm{SCl}$ population meta-analysis. A full color version of this figure is available at the Spinal Cord journal online.

independent risk factor involved in SCI patients that is distinct from the general population. ${ }^{19}$

Much of the research on bladder cancer in SCI patients has focused on determining whether the use of an indwelling catheter results in an increased risk of bladder cancer. Concurrently, evidence emerged suggesting that indwelling catheters were associated with an increased risk of bladder cancer. The prevailing belief is that indwelling catheters, particularly when used for $\geqslant 10$ years, are a risk factor for bladder cancer. ${ }^{21-23}$ Most series of SCI patients with bladder cancer show that a high proportion (50-100\%) used indwelling catheters. ${ }^{5,8,10,11,14,17,18}$ In the most recent study, $>50 \%$ of patients diagnosed with bladder cancer did not have an indwelling catheter. This suggests that the neurogenic bladder, not the indwelling catheter, may be the risk factor for bladder cancer. ${ }^{18}$ West et al. ${ }^{14}$ reported that in the group of SCI bladder cancer patients, those without catheters accounted for $38 \%$ of the bladder tumors, and these patients tended to 


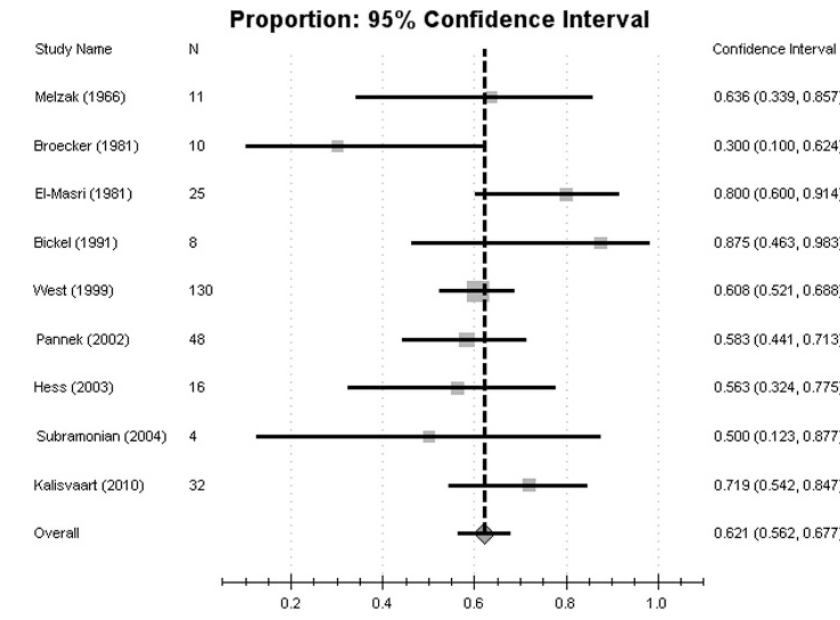

Figure 8 The 1-year overall survival rate after treatment of bladder cancer meta-analysis. A full color version of this figure is available at the Spinal Cord journal online.

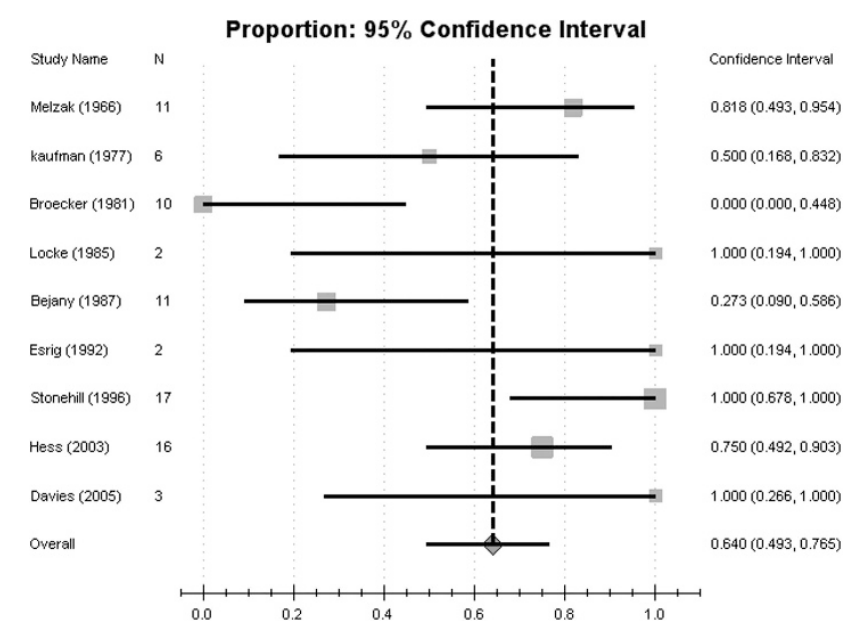

Figure 9 The sensitivity of cystoscopy for detecting bladder cancer metaanalysis. A full color version of this figure is available at the Spinal Cord journal online.

develop bladder cancer more rapidly than their SCI counterparts with indwelling catheters. These conflicting reports are probably compounded by the retrospective nature of the studies, and the fact that bladder management tends to change over a patient's lifetime. Length of indwelling catheterization of the patient population ranged from 6 to 29 years, with an average of 16 years ( $95 \%$ CI, $12-20$ years).

The bladder tumor subtype is another indicator of a unique risk factor among SCI patients. In the general population the estimated frequency of SCC is $2-7 \%{ }^{24,25}$ SCC of the bladder is commonly associated with factors that produce excessive bladder inflammation, such as parasitic infection with schistosomiasis. ${ }^{26,27}$ In the SCI population the estimated percent of SCC is $36.8 \%$ (95\% CI, 31.6-42.5\%). Also, $46.3 \%$ (95\% CI, 33.7-59.4\%) of the bladder cancer histology was traditional TCC. Other pathological subtypes account for $17.1 \%$ (95\% CI, 12.8-22.6\%). SCI-related series of bladder cancer have almost uniformly demonstrated an increased number of SCC tumors $(0-82 \%)$. Pannek ${ }^{16}$ series found that $81 \%$ of the bladder cancer histology was traditional TCC, and Esrig et al. ${ }^{12}$ found

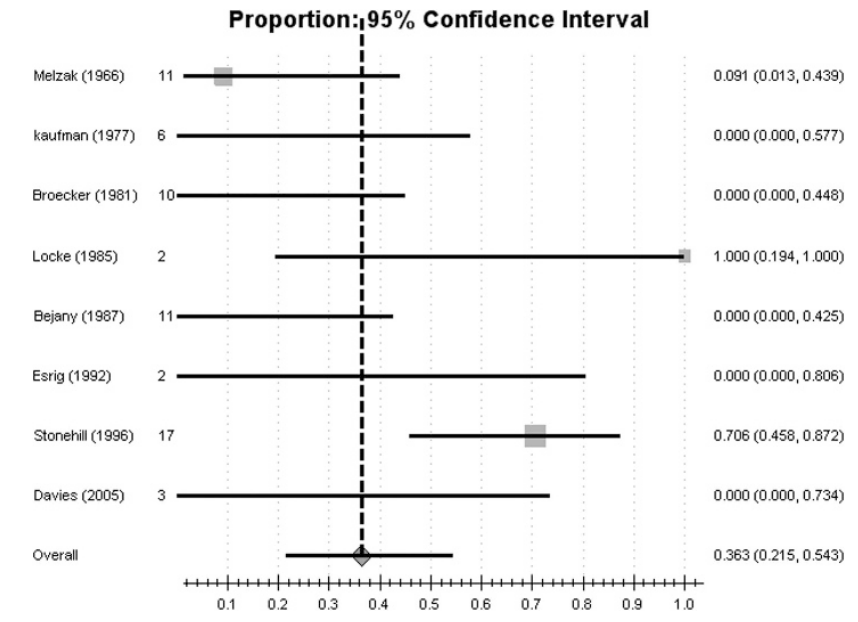

Figure 10 The sensitivity of cytology for detecting bladder cancer metaanalysis. A full color version of this figure is available at the Spinal Cord journal online.

only TCC within his small series. One possible explanation of these findings is that the initial tumor is pathologically TCC that then differentiates into a squamous subtype that is difficult to characterize pathologically. ${ }^{14}$

Bladder cancer-specific mortality has been reported to range from 12 to $57 \% .5,11,15-19$ The 1-year overall survival rate after treatment of bladder cancer was $62.1 \%$ (95\% CI, 56.2-66.7\%). In a well-controlled study by Groah et al.,$^{15}$ the standardized mortality ratio was 71 -fold higher than that of the general population. A study by West et al. ${ }^{14}$ reported a 1-year overall survival rate after treatment of bladder cancer of $61 \%$; similar results $(70 \%)$ were found after 1 year by Stonehill et al. ${ }^{5} \mathrm{Nahm}$ et al..$^{20}$ used the largest SCI cohort in the United States to date, including 566532 person-years of follow-up between 1960 and 2009. They identified 10575 deaths and categorized 99 deaths from bladder cancer. The expected number of deaths from bladder cancer would have been 14.8 if patients with SCI had the same bladder cancer mortality as the general population. This study demonstrated that given the same age, gender and race/ethnicity, patients with SCI are $\sim 6.7$ times (95\% CI, 5.4-8.1) more likely to die of bladder cancer than the general population. ${ }^{20}$

When examining bladder cancer among SCI patients, physicians use the tools (such as urinalysis, urine cytology, cystoscopy and random bladder biopsies) to detect bladder cancer in this population, and to determine whether there is a role for screening with any of these investigations. In the general population, screening cystoscopies are the standard of care after an initial diagnosis of noninvasive bladder cancer. Many series have advocated the use of cystoscopy as an annual screening tool in specific groups of SCI patients. $8,10,12,14,18$ Others have suggested yearly cytology. ${ }^{9}$ However, some studies have shown that screening did not lead to an earlier diagnosis for patients who were later diagnosed with bladder cancer. ${ }^{23,28}$ SCI patients who survived their diagnosis of bladder cancer actually had less screening cystoscopies performed than those who died from the disease. ${ }^{28}$ In this study, cytology demonstrated a sensitivity of $36.3 \%$ (95\% CI, 21.5-54.3\%) for detecting bladder cancer; cystoscopy demonstrated a sensitivity of 64\% (95\% CI, $49.3-76.5 \%)$ for detecting bladder cancer. These screening tests do not fulfill many of the criteria of a screening test: the incidence of bladder cancer in this population is relatively small, bladder cancer is not as easy to detect using conventional tools (owing to the abnormal appearance and histological changes of the SCI 
bladder) and bladder cancer can occur rapidly, making yearly screening less likely to detect the disease at an earlier stage. Therefore, the optimum bladder cancer screening method has not been defined. ${ }^{21}$

The majority of these reports did not calculate age- or gender-adjusted incidence rates, or express their results in patientyear units in order to take into account variable periods of follow-up. Because the tumor is rare in patients with SCI, it would be difficult to conduct well-controlled prospective studies to gain an objective conclusion. As mentioned in Welk et al. ${ }^{19}$ our study was limited by the fact that its conclusions were based on the analysis of limited reports. In a situation where it is difficult to gather a sufficient number of cases, even across multiple institutions, our results could provide important insights into this extremely rare condition based on the meta-analysis. It is important to reflect upon the heterogeneity of the presented data and it is furthermore needed to point out that the biggest share of the evaluated studies are $>10$ years old. In addition, these case series represent an era with a different approach to the management of neurogenic bladder patients.

\section{CONCLUSIONS}

Bladder cancer is a significant oncologic complication in SCI patients. The literature suggests that bladder cancer occurs more often in SCI patients than that in the general population, and is associated with significant mortality. Patients with a long-standing SCI or indwelling catheter usage for 16 years may be at the highest risk for bladder cancer. Further research is required to assess independent risk factors for bladder cancer among SCI patients in order to identify high-risk populations for potential surveillance Strategies.

\section{DATA ARCHIVING}

There were no data to deposit.

\section{CONFLICT OF INTEREST}

The authors declare no conflict of interest.

1 Torre LA, Bray F, Siegel RL, Ferlay J, Lortet-Tieulent J, Jemal A. Global cancer statistics, 2012. CA Cancer J Clin 2015; 65: 87-108.

2 Silverman DT, Hartge P, Morrison AS, Devesa SS. Epidemiology of bladder cancer. Hematol Oncol Clin North Am 1992; 6: 1-30.

3 Howlader N, Noone AM, Krapcho M, Garshell J, Miller D, Altekruse SF et al. SEER Cancer Statistics Review, 1975-2011, National Cancer Institute. Bethesda, MD http://seer.cancer.gov/csr/1975_2011/ based on November 2013 SEER data submission, posted to the SEER website, April 2014.

4 Melzak J. The incidence of bladder cancer in paraplegia. Paraplegia 1966; 4: 85-96.

5 Stonehill WH, Dmochowski RR, Patterson AL, Cox CE. Risk factors for bladder tumors in spinal cord injury patients. J Urol 1996; 155: 1248-1250.

6 Kaufman JM, Fam B, Jacobs SC, Gabilondo F, Yalla S, Kane JP et al. Bladder cancer and squamous metaplasia in spinal cord injury patients. J Urol 1977; 118: 967-971.

7 El-Masri WS, Fellows G. Bladder cancer after spinal cord injury. Paraplegia 1981; 19 : 265-270.

8 Broecker $\mathrm{BH}$, Klein FA, Hackler RH. Cancer of the bladder in spinal cord injury patients. J Urol 1981; 125: 196-197.

9 Locke JR, Hill DE, Walzer Y. Incidence of squamous cell carcinoma in patients with long-term catheter drainage. J Urol 1985; 133: 1034-1035.

10 Bejany DE, Lockhart JL, Rhamy RK. Malignant vesical tumors following spinal cord injury. J Urol 1987; 138: 1390-1392.

11 Bickel A, Culkin DJ, Wheeler JS Jr. Bladder cancer in spinal cord injury patients. J Urol 1991; 146: 1240-1242.

12 Esrig D, McEvoy K, Bennett CJ. Bladder cancer in the spinal cord-injured patient with long-term catheterization: a casual relationship? Semin Urol 1992; 10 102-108.

13 Vereczkey ZA, Schmeidler J, Binard JE, Bauman WA. Bladder cancer risk in patients with spinal cord injury. J Spinal Cord Med 1998; 21: 230-239.

14 West DA, Cummings JM, Longo WE, Virgo KS, Johnson FE, Parra RO. Role of chronic catheterization in the development of bladder cancer in patients with spinal cord injury. Urology 1999; 53: 292-297.

15 Groah SL, Weitzenkamp DA, Lammertse DP, Whiteneck GG, Lezotte DC, Hamman RF. Excess risk of bladder cancer in spinal cord injury: evidence for an association between indwelling catheter use and bladder cancer. Arch Phys Med Rehabil 2002; 83: 346-351.

16 Pannek J. Transitional cell carcinoma in patients with spinal cord injury: a high risk malignancy? Urology 2002; 59: 240-244.

17 Subramonian K, Cartwright RA, Harnden P, Harrison SC. Bladder cancer in patients with spinal cord injuries. BJU Int 2004; 93: 739-743.

18 Kalisvaart JF, Katsumi HK, Ronningen LD, Hovey RM. Bladder cancer in spinal cord injury patients. Spinal Cord 2010; 48: 257-261.

19 Welk B, Mclntyre A, Teasell R, Potter P, Loh E. Bladder cancer in individuals with spinal cord injuries. Spinal Cord 2013; 51: 516-521.

20 Nahm LS, Chen Y, DeVivo MJ, Lloyd LK. Bladder cancer mortality after spinal cord injury over 4 decades. J Urol 2015; 193: 1923-1928.

21 Cameron AP, Rodriguez GM, Schomer KG. Systematic review of urological followup after spinal cord injury. J Urol 2012; 187: 391-397.

22 Navon JD, Soliman H, Khonsari F, Ahlering T. Screening cystoscopy and survival of spinal cord injured patients with squamous cell cancer of the bladder. J Urol 1997; 157: 2109-2111.

23 Stonehill WH, Goldman HB, Dmochowski RR. The use of urine cytology for diagnosing bladder cancer in spinal cord injured patients. J Urol 1997; 157: 2112-2114.

24 Hess MJ, Zhan EH, Foo DK, Yalla SV. Bladder cancer in patients with spinal cord injury. J Spinal Cord Med 2003; 26: 335-338.

25 Manunta A, Vincendeau S, Kiriakou G, Lobel B, Guillé F. Non-transitional cell bladder carcinomas. BJU Int 2005; 95: 497-502.

26 Abol-Enein H. Infection: is it a cause of bladder cancer? Scand J Urol Nephrol Suppl 2008; 218: 79-84.

27 Rosin MP, Anwar WA, Ward AJ. Inflammation, chromosomal instability, and cancer: the schistosomiasis model. Cancer Res 1994; 54 (7 Suppl): 1929s-1933s.

28 Groah SL, Lammertse DP. Factors associated with survival after bladder cancer in spinal cord injury. J Spinal Cord Med 2003; 26: 339-344. 